\title{
Consumo de benzodiacepinas en una muestra de pacientes en Programa de Tratamiento con Derivados Opiáceos (PTDO)
}

\section{Benzodiazepine use in a sample of patients on a treatment program with opiate derivatives (PTDO)}

Ana Ma Fernández Sobrino*; Verónica Fernández RODRÍGUEZ ${ }^{\star \star}$; JOSÉ LÓPEZ CASTRO***
*Psiquiatra. Unidad Asistencial de Drogodependencias de Monforte de Lemos.

** D.U.E. Unidad Asistencial de Drogodependencias de Monforte de Lemos.

${ }^{\star \star \star}$ Doctor en Medicina. Especialista en Medicina Interna. Complexo Hospitalario de Ourense..

Enviar correspondencia a:

Ana $\mathrm{M}^{a}$ Fernández Sobrino.

C/ Otero Pedrayo s/n.27400 Monforte de Lemos (Lugo).

E-mail: uadanamonforte@yahoo.es.

\section{RESUMEN}

Objetivos: Las benzodiacepinas (BZD) son fármacos ampliamente prescritos, pero su utilización es controvertida en pacientes que presentan un trastorno por uso/abuso de sustancias debido al riesgo de tolerancia y abuso/dependencia. El objetivo de este trabajo es determinar la utilización de benzodiacepinas en los pacientes a tratamiento con metadona y compararlo con la literatura existente.

Metodologia: Se ha seleccionado una muestra de 43 pacientes cuyos criterios de inclusión fueron estar en PTDO en la Unidad Asistencial de Drogodependencias (UAD) de Monforte. Se analizaron variables demográficas y de consumo. La recogida de datos y el posterior análisis se ha realizado mediante el programa estadistico SPSS 12.0.

Resultados: El 46,5\% toman benzodiacepinas y de éstos el 60\% es mayor de 45 años. La más usada es alprazolam $(p<0,001)$. El 70\%, toman un único principio activo. El principal presciptor es el médico de cabecera y la indicación más frecuente es el insomnio.

Discusión y conclusiones: Los datos de consumo de BZD y su relación con el tiempo de permanencia en metadona son similares a los de otros estudios publicados. A pesar de la recomendación de utilizar en pacientes con trastorno por abuso de sustancias BZD de vida media larga, resulta llamativo que en nuestro caso la más prescrita sea alprazolam.

Palabras clave: benzodiacepinas, metadona, indicación, prescripción, uso/ abuso.

\section{ABSTRACT}

Objectives: Benzodiazepines (BZ) are widely prescribed drugs, but their use is controversial in patients who present a disorder caused by substance use/abuse, due to the risk of tolerance and abuse/dependence. The purpose of this work is to determine the levels of $B Z$ use in patients treated with methadone and to compare them with findings from the current literature.

Methodology: a sample of 43 patients was selected; inclusion criterion was being on the PTDO (treatment program with opiate derivatives) at the UAD (Drug-dependence unit) in Monforte de Lemos (Lugo-Spain). Demographic and use variables were analyzed. Data collection and subsequent analysis were carried out using the statistical program SPSS 12.0.

Results: $46.5 \%$ of the patients take BZ, and of these, $60 \%$ are over age 45 . The most widely used of these is alprazolam $(p<0.001)$, and $70 \%$ take just one active ingredient. The main prescriber is the general practitioner, and the most frequent indication is insomnia.

Discussion and Conclusions; BL-use data and their relationship to time spent on methadone are similar to those of other published studies. Despite the recommendation to use long half-life BZ in patients with substance abuse disorders, it is striking that in the case studied here the most widely prescribed type is alprazolam.

Key words: benzodiazepines, methadone, indication, prescription, use/ abuse. 


\section{INTRODUCCIÓN}

os pacientes en PTDO (metadona) presentan con frecuencia simultáneamente dependencia de opiáceos y uso de otras sustancias. La experiencia clínica demuestra que este colectivo utiliza benzodiazepinas (BZD) para potenciar el efecto de la metadona, para intoxicarse cuando no disponen de otra sustancia, o como autoprescripción para tratar un trastorno psicopatológico asociado'.

La experiencia clínica dice que más del $60 \%$ de los pacientes a tratamiento con metadona consume regularmente otras drogas, principalmente cannabis, BZD, alcohol y cocaína. Esto constituye un grave problema, con importantes repercusiones pronósticas y terapéuticas ${ }^{1,2}$.

En la práctica diaria de la U.A.D. de Monforte, observamos un "uso" generalizado de BZD entre los pacientes en PTDO. El objetivo del presente trabajo es constatar la veracidad de esta observación y describir las características del consumo.

\section{MATERIAL Y MÉTODOS}

Estudio de prevalencia del consumo de BZD de los pacientes adscritos en la actualidad a tratamiento en PTDO en la U.A.D. de Monforte. Los criterios de selección establecidos han sido amplios debido al reducido volumen asistencial de dicho centro por lo que el único requisito que debían cumplir los pacientes era encontrarse en programa de metadona y dar su consentimiento para participar en el estudio. La sistemática de recogida de datos se realizó a través de la revisión de las Historias Clínicas de donde se extrajeron datos de identificación, prescripción y tiempo de permanencia en metadona. También se realizó una entrevista personal con el paciente por lo que este trabajo exige la colaboración de los mismos. La muestra está formada por 43 usuarios, el total de pacientes en PTDO de los cuales 41 eran hombres y 2 mujeres. La edad media era de 42,1 años (rango 27 a 63 años). La participación fue voluntaria mediante consentimiento verbal y se garantizó el anonimato de los resultados. Se les preguntaba si hacian uso de alguna BZD, cuál, dosis, quién la prescribe, indicación y tiempo de consumo.

Para el análisis de datos se ha utilizado el paquete estadístico SPSS 12.0 para Windows. Para la descripción y extracción de conclusiones se han utilizado porcentajes, medidas de tendencia central, la prueba estadistica de $\chi^{2}$ y la $\mathrm{t}$ de Student para muestras independientes.

\section{RESULTADOS}

Del total de pacientes de la muestra $(N=43)$ se ha constatado el uso de BZD en un 46,51\%. Dentro de este grupo se distinguió entre dos tipos de consumo: ocasional y mantenido ( $>6$ meses sin interrupción). Nos encontramos que el $75 \%$ lo hacen de forma continua $(p<0,001)$.

En cuanto al policonsumo se comprueba que el $70 \%$ de los pacientes que usan BZD utilizan un principio activo frente al 30\% que consumen dos o más. Todos los casos en los que hay consumo múltiple de BZD existe continuidad. A pesar de no resultar estadisticamente significativo es un dato llamativo.

Los principios activos más utilizados $(p<0,001)$ en orden decreciente son: alprazolam (65\%), lormetacepam, flunitracepam y bromacepam con un $10 \%$ de aparición cada uno y clonacepam (5\%). Cuando existe policonsumo la asociación más frecuente es alprazolam/flunitracepam $(\mathrm{p}=$ 0,091 ).

En la muestra estudiada la utilización de BZD era por ansiedad el $40 \%$ y por insomnio el $60 \%$. Cuando este dato lo analizamos en función de la edad se aprecia una distribución etaria del motivo del consumo, en los pacientes de mayor edad es el insomnio el que lo motiva en un 75\% (Figura 1).

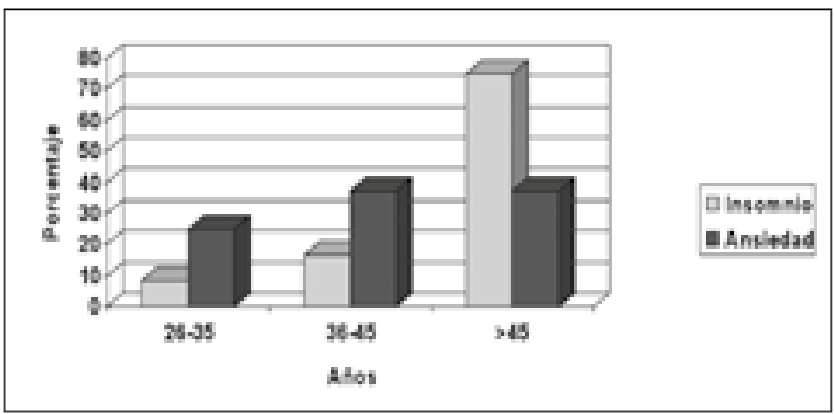

Figura 1. Distribución de la indicación por grupo etario.

Entre el tiempo de permanencia en PTDO y el consumo de BZD existe una asociación estadísticamente significativa $(\mathrm{p}<$ $0,05)$. Se establecieron cuatro intervalos de tiempo: $<1$ 1año, entre 1 y 5 , entre 5 y 10 y $>10$ años. Del total de la muestra el 30\% lleva más de 10 años en PTDO. Se comprobó que cuanto más tiempo llevan tomando metadona el consumo de BZD es más frecuente (Figura 2).

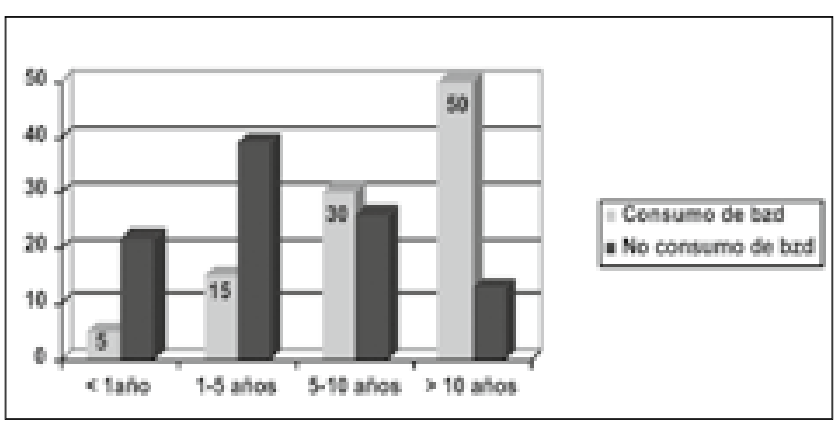

Figura 2. Relación entre tiempo en metadona y consumo de BZD (\%).

Otro parámetro estudiado ha sido la dosis de metadona prescrita en relación con el consumo de BZD. El resultado de esta asociación es que en el grupo de consumidores de BZD 
la dosis media de metadona es más elevada. Sin embargo no es estadisticamente significativo.

Otra de las variables analizadas ha sido el origen de la prescripción para determinar si prevalece el autoconsumo o por el contrario existe una prescripción facultativa que avale los tratamientos. Casi la totalidad de la muestra obtiene las BZD con receta médica. El principal prescriptor es el médico de atención primaria (70\%), seguido de otros especialistas, generalmente internistas, $(15 \%)$ y el facultativo de salud mental (10\%). Sólo fue detectado un caso en que el consumo se produce sin prescripción $(p<0,001)$.

Otro aspecto importante a tener en cuenta es la intención de abandono del consumo, bien sea a corto o a largo plazo. En la muestra analizada el $85 \%$ no se planteaba la posibilidad de dejar de consumir BZD, sólo un 10\% manifestó su deseo de abandonarlas y el 5\% presenta dudas.

Del total de la muestra un tercio es VIH + , y dentro de este grupo el 64\% consume BZD. De los dos tercios restantes de la muestra, solamente el $37,9 \%$ son consumidores ( $p=$ 0,104).

\section{DISCUSIÓN}

El porcentaje de pacientes que consumen BZD en nuestra muestra es de $46,51 \%$, este dato es superior al encontrado en estudios publicados actualmente en donde se refiere un consumo de BZD del $35 \%{ }^{3}$ y otros en los que se analiza el consumo de hipnosedantes en general y que arrojan cifras en torno al $25 \%{ }^{4}$. No así en otros trabajos donde el porcentaje de consumidores de BZD fue del 71\% (36\% ocasionales; 35\% regulares) ${ }^{5}$. Estos porcentajes podrían ser superiores debido a los frecuentes falsos negativos en el caso del alprazolam ya que los metabolitos de este fármaco excretados en orina se encuentran en concentraciones menores de las requeridas para su detección en los análisis rutinarios ${ }^{2}$.

En contraposición a estudios publicados no se encontró consumo de diacepam ni oxacepam ${ }^{2,6}$.

La frecuencia del uso de las BZD también se ve reflejado en el aumento en los últimos años de las desintoxicaciones hospitalarias de esta sustancia en pacientes en PTDO, tal y como refleja el reciente estudio de 0 choa $E$ et al. donde también se hace referencia a las dificultades añadidas a las desintoxicaciones de metadona cuando el paciente presenta consumo concomitante de BZD. ${ }^{7}$

Según algunos autores el tiempo que los pacientes llevan tomando metadona parece ser independiente del consumo de $B Z D^{8}$ otros trabajos sin embargo ofrecen resultados en la misma linea ${ }^{5}$ que el nuestro donde la asociación de estos dos datos resulta estadisticamente significativa a favor de que al aumentar la permanencia en el PTDO la frecuencia del consumo de BZD va aumentando gradualmente. No hemos podido constatar si todos los pacientes de la muestra consumian BZD al inicio del PTDO debido a que algunas historias clínicas eran muy antiguas y no disponian de este dato.
Hay estudios que sugieren que cuanto mayor es la dosis de metadona mayor es el número de pacientes que consumen $\mathrm{BZD}^{1,2}$. En nuestro caso no ha sido posible demostrarlo por el reducido número de la muestra.

En la mayoría de los manuales y guías clínicas se recomiendan como fármacos de primera elección para el trastorno de ansiedad las BZD, pero cuando se trata de pacientes diagnosticados de trastorno por uso de sustancias deberian agotarse otros recursos farmacológicos (ISRS, antipsicóticos.... $)^{9,10}$ y no farmacológicos para el tratamiento de la ansiedad/insomnio debido al riesgo que existe de abuso/dependencia de las mismas. Cuando en este tipo de pacientes se requiere tratamiento con BZD se evitarán las de alta potencia y semivida corta, como el alprazolam. Las más aconsejadas serían aquellas de semivida larga como el diacepam, el ketazolam, el cloracepato o el clonacepam y con prescripción controlada ${ }^{9,10}$. En contraposición a las recomendaciones un amplio porcentaje de la muestra consumen aquellas BZD menos indicadas y sin embargo las más aconsejadas no figuran entre los principios activos más consumidos.

Más del $50 \%$ de los pacientes que consumen BZD lo hacen por insomnio lo que nos lleva a plantearnos la posibilidad de utilizar otro tipo de medidas alternativas 0 complementarias al tratamiento farmacológico, entre ellas la psicoterapia y los talleres de higiene del sueño para adquirir hábitos de vida más saludables y reducir o suprimir los fármacos.

La bibliografía que hace referencia a la intención de abandono de consumo de BZD en PTDO es de hace una década aproximadamente por lo que no hemos considerado pertinente la comparación con estos estudios ya que ni el tipo de BZD consumida es el mismo" ${ }^{11}$.

Ningún paciente del estudio demandó desintoxicación de BZD lo que es coherente con que el $85 \%$ no tienen intención de dejarlas. En nuestra Unidad hasta el año 2006 no se registró ninguna demanda de desintoxicación de BZD ${ }^{12}$. Afortunadamente cada día son menos los usuarios que se infectan con el VIH a través del consumo de drogas, en estos momentos sólo un tercio de los pacientes en PTDO tienen serología positiva para el VIH. Relacionando este dato con el objetivo del estudio encontramos que más del 60\% de los infectados consume BZD por el contrario es más del 60\% de los no infectados los que no consumen. Extraer más conclusiones de este dato es controvertido por la relación que existe entre el mayor tiempo de permanencia en PTDO y el status $\mathrm{VIH}+$.

\section{AGRADECIMIENTOS}

Los autores agradecen a los usuarios/as que han participado ya que sin sus aportaciones y su amable y desinteresada colaboración este trabajo no habría sido posible. 


\section{REFERENCIAS}

1. Díaz González T. Uso concomitante de drogas en los PMMs: prevalencia e importancia pronóstica. En: Fernández Miranda JJ, Marina González PA. Manual de los tratamientos de mantenimiento con derivados opiáceos. Oviedo: Papiro, 1999; 191-279.

2. Micó JA, Moreno Brea MR, Rojas Corrales O, GibertRahola J. Interacciones medicamentosas en programas de mantenimiento y deshabituación a opiáceos. V Encuentro Nacional sobre drogodependencia y su enfoque comunitario. Chiclana de la Frontera, 11-13 de Marzo de 1998.

3. Gimeno C, Esteban J, Pellin MC, Climent JM y Grupo de investigación en adicciones de Alicante. Mejoría de la gravedad de la adicción en los tratamientos con metadona. Adicciones 2002; 14: 151-60.

4. March Cerdá JC, Martín-Ruiz E, Oviedo-Joekes E, Rivadeneira Sicilia A, Rodríguez Reinado C. Percepción de usuarios de los Programas de Tratamiento con Metadona sobre la accesibilidad y atención recibida de Ios equipos terapéuticos. Adicciones 2006; 18: 359-70.

5. Brands B, Blake J, Marsh DC, Sproule B, Jeyapalan R, Li $S$. The impact of benzodiazepine use on methadone maintenance treatment outcomes. J Addict Dis. 2008; 27: 37-48.

6. Bramness JG, Kornør H. Benzodiazepine prescription for patients in opioid maintenance treatment in Norway. Drug Alcohol Depend. 200790 (2-3):203-9.
7. Ochoa E, Salvador E, Madoz-Gúrpide A, Lázaro M. Desintoxicación de dosis elevadas de metadona en una Unidad de Desintoxicación Hospitalaria (UDH). Experiencia clínica. Adicciones 2008; 20: 245-50.

8. Swensen G, Ilett KF, Dusci LJ, Hackett LP, Ong RT, Quigley AJ et al. Patterns of drugs use by participants in the Western Australian methadone program, 19841991. Med J Aust 1993; 159: 373-6.

9. Fernández Miranda JJ, Pereiro Gómez C. Comorbilidad psiquiatrica en la dependencia de opiáceos. En: Fernández Miranda JJ. Guía clínica para el tratamiento de la dependencia de opiáceos. Valencia: Socidrogalcohol, 2007; p. 111-6.

10. Montes Pérez M, Baño Rodrigo D, Jiménez García JM, Otero Lamas F, Ruiz de Apodaka J, Sánchez Vázquez R. Programas de mantenimiento con agonistas opiaceos. En: Fernández Miranda JJ. (Coordinador) Actuaciones clínicas en trastornos adictivos. Barcelona: Aula Médica, 2002; p. 303-36.

11. Gelkopf M, Bleich A, Hayward R, Bodner G, Adelson M. Characteristics of benzodiazepine abuse in methadone maintenance treatment patients: a 1 year prospective study in a Israeli clinic. Drug Alcohol Depend, 1999; 55: 63-8.

12. Fernández Rodríguez $V$, Fernández Sobrino $A M$, López Castro J. Pasado y presente. ¿Hay diferencia en el perfil del usuario de la Unidad Asistencial de Drogodependencias de Monforte? 1996-2006. Trastornos Adictivos. 2008; 10: 121-6. 